\section{MS5-P37 Structural investigation of the small GTPase ARL4D}

Sasa Petrovic ${ }^{1}$, Yvette Roske ${ }^{1}$, Udo Heinemann ${ }^{1}$

1. Max Delbrück Center for Molecular Medicine, Robert-Rössle-Straße 10, 13125 Berlin, Germany

email: sasa.petrovic@mdc-berlin.de

ARL4D (ADP-ribosylation factor-like protein 4D) is a small GTPase which cycles from the membrane-attached GTP-bound form and the cytosolic GDP-bound form (1). It is a member of the ADP-ribosylation factor/ARF-like protein (ARF/ARL) family of Ras-related GTPases. ARL4D contains a nuclear localization signal on the C-terminus, thus can be found in the nucleus (2), as well as at the cytoplasm and plasma membrane (1). The membrane binding of ARL4D is mediated by both an $\mathrm{N}$-terminal myristoyl group and an N-terminal amphipathic helix (3). ARLAD expression is developmentally regulated during embryogenesis (2), and its overexpression has been shown to suppress adipogenesis (4). ARL4D has the ability to recruit ARNO (Arf nucleotide-binding site opener) to the plasma membrane through interaction with its pleckstrin homology (PH) domain (1). Subsequently, ARNO acts as a nucleotide exchange factor and activator of Arf6, a small GTPase that regulates endocytosis, actin dynamics and cell adhesion $(5,6)$. Our aim is to characterize, structurally and biochemically, ARL4D alone, and in complex with its interaction partner ARNO. This will provide an insight into the mode of function of this GTPase, and a possibility to explain specific features compared to other members of the ARF/ARL family.

(1) Hofmann E, et al. (2007). "The Arl4 family of small $\mathrm{G}$ proteins can recruit the cytohesin Arf6 exchange factors to the plasma membrane". Current Biology, 17: 711-716.

(2) Lin CY, et al. (2000). "ARL4, an ARF-like protein that is developmentally regulated and localized to nuclei and nucleoli”. Journal of Biological Chemistry, 275: 37815-37823.

(3) Pasqualato S, et al. (2002). "Arf, Arl, Arp and Sar proteins: A family of GTP-binding proteins with a structural device for 'front-back' communication". EMBO Reports, 3: 1035-1041.

(4) Yu J, et al. (2011). "Overexpression of the small GTPase Arl4D suppresses adipogenesis". International Journal of Molecular Medicine, 28: 793-798.

(5) Chardin P, et al. (1996). "A human exchange factor for ARF contains Sec7- and pleckstrin-homology domains". Nature, 384: 481-484.

(6) Li C, et al. (2007). "ARL4D recruits cytohesin-2 / ARNO to modulate actin". Molecular Biology of the Cell, 18: 4420-4437.

Keywords: crystallography, GTPase

\section{MS5-P38 Characterization of electrostatic} interactions in active sites of caspases

Urszula A. Budniak ${ }^{1}$, Paulina M. Dominiak ${ }^{1}$

1. Department of Chemistry, University of Warsaw, Pasteura 1, 02-093 Warsaw, Poland

email: ubudniak@chem.uw.edu.pl

Apoptosis (programmed cell death) is an intensely examined cell process because its failure can trigger a tumor development. Enzymes that play crucial role in apoptosis, but also in necrosis and inflammation, are called caspases and belong to the family of cysteine proteases. Initiator caspases receive a signal, get activated and cleave effector caspases, which cleave other proteins leading to cell death. This chain reaction is known as "caspase cascade".

For the purpose of this project various caspases interacting with inhibitors were examined. The Protein Data Bank $[1,2]$ was utilized to find the highest quality structures of caspases bounded up with ligands. After structure validation, all missing hydrogen atoms were added. Electron density distribution of protein complexes was reconstructed using the Transferred Aspherical Atom Model approach based on the University at Buffalo Databank [3,4]. This database contains multipolar parameters describing electron density of pseudoatoms obtained via theoretical calculations. On the basis of electron density distribution, energy of electrostatic interactions was calculated. Characterization of intermolecular interactions was possible thanks to calculations in the Mopro [5] or XD [6] program.

Investigations of energetic properties of caspases may contribute to our knowledge about protein properties in general and, more importantly, gives an opportunity to find novel ligands binding more effectively to their actives sites, what is essential for drug design.

References:

1. RCSC PDB www.rcsb.org/pdb.

2. Berman, H.M et al. (2000) Nucl. Acids Res., 28: 235-242.

3. Volkov, A., Li, X., Koritsanszky, T. S., Coppens, P. (2004) J. Phys. Chem. A, 108: 4283-4300.

4. Jarzembska, K. N., Dominiak, P. M. (2010) Acta Cryst A, 68: 139-147.

5. Guillot B., Viry L., Guillot R., Lecomte C. \& Jelsch C. (2001) J. Appl. crystallogr., 34: 214-223.

6. Volkov, A.; Macchi, P.; Farrugia, L. J.; Gatti, C.; Mallinson, P.; Richter, T.; Koritsanszky, T. (2006) XD2006 - a computer program package for multipole refinement, topological analysis of charge densities and evaluation of intermolecular energies from experimental and theoretical structure factors.

7. DOI: $10.2210 / \mathrm{pdb} 4 \mathrm{ps} 1 / \mathrm{pdb}$. 


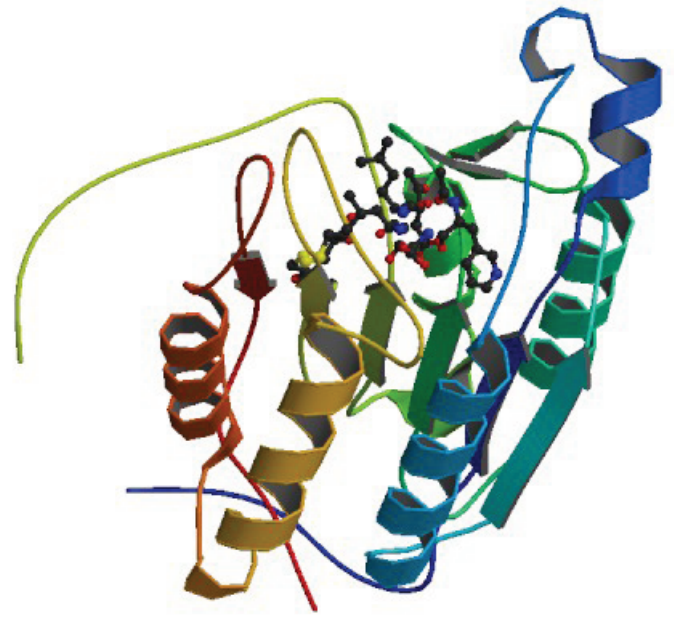

Figure 1. An example of caspase with a ligand (PDB ID: 4PS1) [7]

Keywords: caspases, ligands, electrostatic interactions, UBDB, TAAM

\section{MS5-P39 Crystallization and structural characterization of glyceraldehyde dehydrogenase from Thermoplasma acidophilum}

Iuliia Iermak ${ }^{1}$, Jeroen R. Mesters ${ }^{2}$, Oksana Degtjarik ${ }^{1}$, Fabian Steffler ${ }^{3}$, Volker Sieber ${ }^{3}$, Ivana Kuta Smatanova ${ }^{1,4}$

1. Faculty of Science, University of South Bohemia, Branišovska 31, CZ-37005 Česke Budejovice, Czech Republic

2. Institute of Biochemistry, University of Lübeck, Ratzeburger Allee 160, 23538 Lübeck, Germany

3. Chemistry of Biogenic Resources, Straubing Centre of Science, Technische Universität München, Schulgasse 16, 94315 Straubing, Germany

4. Institute of Nanobiology and Structural Biology GCRC, Academy of Sciences of the Czech Republic, Zamek 136, 37333 Nove Hrady, Czech Republic

email: julia.ermak90@gmail.com

Biotechnological production of chemical compounds is an environmentally more gentle process compared to their fabrication from natural fossil resources. In terms of bio-production, cell-free processes are more effective than microbial production techniques since the enzymes can tolerate higher concentrations of final product than the cells. The glyceraldehyde dehydrogenase from Thermoplasma acidophilum (TaAlDH) is a part of an artificial cell-free enzyme cascade for production of isobutanol and ethanol from glucose. TaAlDH catalyzes the oxidation of D-glyceraldehyde to D-glycerate in this synthetic pathway. Various mutants of TaAlDH were constructed by random approach followed by site-directed and saturation mutagenesis in order to improve the enzymes' properties essential for its functioning within the cascade. Further optimization of $\mathrm{TaAlDH}$ requires structural information about the enzyme for which crystallization followed by X-ray diffraction analysis was employed.

Different types of TaAlDH wild-type crystals grew within one to two weeks after initial screening in 30 diverse conditions. In order to obtain the best quality crystals, optimization was carried out considering the following parameters: (a) already known diffraction quality of crystals; $(b)$ size and shape of crystals (big single crystals with sharp edges preferred); $(c)$ different crystal forms. Optimization, including variation of $\mathrm{pH}$, protein and precipitant concentrations and ratios, resulted in adequate crystal quality only for condition H6 of the Morpheus screen (Molecular Dimensions Ltd., UK). These crystals diffracted X-rays to $1.95 \AA$ resolution and belonged to space group $\mathrm{P} 2{ }_{1}$ with 8 molecules per asymmetric unit and unit cell parameters of $a=95.29 \AA$, $b=152.35 \AA, c=149.90 \AA, \alpha=\gamma=90.0^{\circ}, \beta=92.19^{\circ}$.

The structure of TaAlDHwt was solved by molecular replacement using the coordinates of betaine-aldehyde dehydrogenase from Pseudoalteromonas atlantica T6c (sequence identity $38 \%$, PDB ID $3 \mathrm{~K} 2 \mathrm{~W}$ ). The final model contains two tetramers in the asymmetric unit that are related by non-crystallographic symmetry with 